\title{
Low Stratospheric Wind Measurement Using Mobile Rayleigh Doppler Wind LIDAR
}

\author{
Zhi-feng Shu ${ }^{1}$, Xian-kang Dou ${ }^{1 *}$, Hai-yun Xia ${ }^{1}$, Dong-song Sun ${ }^{1}$, Yan Han ${ }^{1}$, Hyunki Cha ${ }^{2}$, \\ Dukhyeon Kim ${ }^{3}$, Guo-cheng Wang ${ }^{1}$, Sunghoon Baik ${ }^{2}$, and Dong-dong $\mathrm{Hu}^{1}$ \\ ${ }^{I}$ School of Space and Earth Science, University of Science and Technology of China, \\ Hefei 230026, P. R. China \\ ${ }^{2}$ Korea Atomic Energy Research Institute, Daejeon 305-353, Korea \\ ${ }^{3}$ Hanbat National University, Daejeon 305-719, Korea
}

(Received December 22, 2011 : revised March 22, 2012 : accepted March 29, 2012)

\begin{abstract}
A mobile Rayleigh Doppler wind LIDAR at an eye-safe wavelength of $355 \mathrm{~nm}$ incorporating double-edge technique with triple-channel Fabry-Perot etalon is developed for wind measurement from 5 to $40 \mathrm{~km}$. The structure of this LIDAR system is described. An intercomparsion experiment with rawinsonde is made, showing good agreement with expected measurement accuracy. A continuous observation of stratosphere wind field for several days with temporal resolution of $15 \mathrm{~min}$ and spatial resolution of $200 \mathrm{~m}$ from 5 to $40 \mathrm{~km}$ is presented, demonstrating the stability and robustness of the LIDAR. A stratospheric quasi-zero wind layer can be found at around $20 \mathrm{~km}$ with a direction change from east to west evident in the continuous observation.
\end{abstract}

Keywords: Wind LIDAR, Observation, Fabry-Perot etalon, Rayleigh scattering

OCIS codes : (120.0120) Instrumentation, measurement, and metrology; (140.0140) Lasers and laseroptics; (280.0280) Remote sensing and sensors

\section{INTRODUCTION}

Developing accurate active remote sensors for global wind measurements has long been a high priority need for climate studies, improving current meteorological modeling and weather prediction and other dynamic processes [1-4]. A Mie Doppler wind LIDAR was already successfully developed at Anhui Institute of Optics and Fine Mechanics, CAS, in 2006. This aerosol-based wind LIDAR can be used only for wind measurement in the low troposphere where the aerosol density is thick and Mie backscatter is strong [5-7]. For the high altitude where the aerosol concentration is extremely thin [8], this kind of wind LIDAR is not valid. Fortunately, atmospheric molecules are a dependable and reasonably uniform source on a global basis. For the purpose of wind measurement at high altitude, such as the low stratosphere, a Doppler wind LIDAR based on Rayleigh backscatter by atmospheric molecules is being built in China.

\section{WIND MEASUREMENT PRINCIPLE}

The frequency discriminator for Doppler shift detection in the LIDAR system is a triple channel Fabry-Perot etalon with two edge channels located in the wings of the broadened molecular backscattered spectrum to determine the Doppler shift, as shown in Fig. 1. The locking channel of the etalon is used to measure the outgoing laser frequency and locks the etalon cavity to the laser frequency for a high accuracy wind measurement. The frequency of transmitted laser will be located at the cross point of the two transmission curves corresponding to the two edge channels. The atmospheric molecular backscattered signal received carries a frequency shift from the outgoing laser. Therefore there will be a difference in the relative intensities from the outgoing laser after the backscattered signal passes the two edge channel etalon. The hatched areas in Fig. 1 stand for the received signal relative intensities that are the convolutions of the edge functions and the backscattered signal spectra. The blue area is greater than the green part, indicating a laser

\footnotetext{
*Corresponding author: dou@ustc.edu.cn

Color versions of one or more of the figures in this paper are available online.
} 


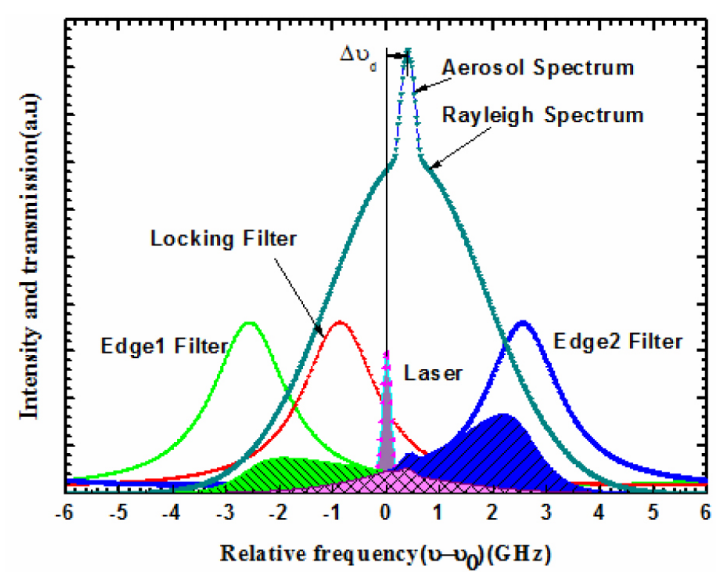

FIG. 1. Spectral profiles of Rayleigh and Mie backscattering and transmission of three filters.

Doppler frequency shift in the received signal, reducing one edge channel of the etalon received signal intensity and increasing the other. So the Doppler shift frequency $\Delta v_{d}$ can be deduced from the signal difference and transmission curve. The radial wind velocity can be expressed as a function of the Doppler shift as

$$
u=\frac{\lambda}{2} \Delta v_{d}
$$

Where $\lambda$ is the emitted laser frequency, $u$ is the wind speed on the line of sight.

\section{LIDAR SYSTEM DESCRIPTION}

The development of the mobile Rayleigh Doppler wind LIDAR for wind measurement is based on the Rayleigh backscatter. By utilizing the molecular backscatter, it could obtain the wind profile up to the stratosphere. The details of the LIDAR system configuration have been described elsewhere [9-11] and will be briefly reviewed here. The system consists of four major modules, which are the laser transmitter, the scanning telescope (transceiver), the receivers and the controlling system, as shown in Fig. 2. All the modules are fixed on a truck. The laser is an injection seeded, flashlamp pumped Nd:YAG laser which produces an output laser beam at $354.7 \mathrm{~nm}$ with a repetition rate of 30 $\mathrm{Hz}$. The maximum laser pulse energy is typically in the range of $300 \sim 400 \mathrm{~mJ}$ at $354.7 \mathrm{~nm}$ for the Rayleigh Doppler wind measurements. A $45 \mathrm{~cm}$ aperture scanner is mounted on the platform of the truck to allow access to the atmosphere. This matching azimuth-over-elevation scanner can provide full hemispherical pointing. Most of the light from the transmitter is expanded by a $10 \mathrm{X}$ expander to compress the beam divergence, and then transmitted to the atmosphere by the two-dimensional scanner. Then the backscattered signal that carries Doppler-shift information is collected by

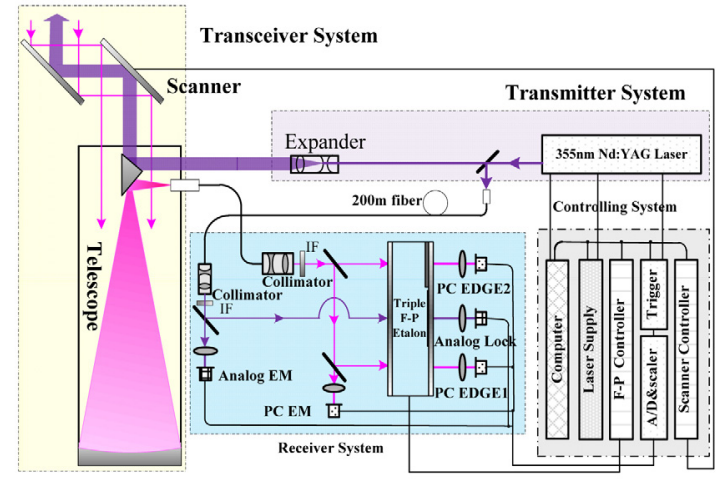

FIG. 2. Schematic diagram of Rayleigh Doppler LIDAR system. (IF - interference filters, PC - photon counting detector, EM - energy monitor)

TABLE 1. Rayleigh Doppler LIDAR parameters

\begin{tabular}{l|l}
\hline \multicolumn{1}{c|}{ Parameter } & \multicolumn{2}{c}{ Value } \\
\hline Transmitter & $354.7 \mathrm{~nm}$ \\
Wave length & $200 \mathrm{MHz}$ \\
Laser line width & $400 \mathrm{~mJ}$ \\
Laser energy/pulse & $30 \mathrm{~Hz}$ \\
Laser repetition rate & \\
\hline Transceiver & $45 \mathrm{~cm}$ \\
Telescope aperture & $0.2 \mathrm{mrad}$ \\
Field of view & $>85 \%$ \\
Optical efficiency & $360^{\circ} \times 90^{\circ}$ \\
Scan range & $30^{\circ}$ \\
Zenith angle & \\
\hline Receiver & $12 \mathrm{GHz}$ \\
Etalon FSR & $1.7 \mathrm{GHz}$ \\
Etalon FWHM & $5.1 \mathrm{GHz}$ \\
Edge channel separation & $1.7 \mathrm{GHz}$ \\
Locking separation & $60 \%$ \\
Etalon peak transmission & $21 \%$ \\
CPM quantum efficiency & $0.15 \mathrm{~nm}$ \\
Filter bandwidth & $>40 \%$ \\
Filter peak transmission & \\
\hline
\end{tabular}

telescope and then it is coupled into $200 \mu \mathrm{m}$ diameter multimode fiber from the telescope to the receiver. Before that, a very small fraction of the outgoing laser is coupled directly into the receiver through a $100 \mu \mathrm{m}$ diameter multimode fiber, and used as the reference signal to determine its frequency and to lock the etalon to the laser frequency.

In the receiver, the reference light and the backscattered light are collimated, and then pass through interference filters (IF) with the bandwidth of $0.15 \mathrm{~nm} @ 355 \mathrm{~nm}$. Then, the backscattered light is split equally into two edge channels. $23 \%$ of the reference light and $6 \%$ of the backscattered light are used monitor the signal intensities. The $77 \%$ reference light illuminating the locking channel of the triple Fabry-Perot etalon is used to measure the outgoing 
laser frequency relative to the etalon. The Doppler shift information is retrieved from the transmission changes of the backscattered signal on the two edge channels. The two reference light channels are strong enough to be detected by PMT detectors in analog mode. While the backscattered light channels are detected in photon counting mode using photomultipliers. The laser operation, FPI spacing parallelism, data collection, and X-Y scanner orientation are controlled by an industrial computer. The system parameters are summarized in Table 1.

\section{INTERCOMPARSION EXPERIMENT AND WIND MEASUREMENT}

The mobile Rayleigh Doppler wind LIDAR system began wind measurement in Dec. 2009. A validation experiment was carried out at Urumqi, Xin Jiang province by a field observation mission. The wind velocity and direction derived from the wind LIDAR are compared with the data obtained from the rawinsonde in Aug. 2010.

An example of signals from the two edge channels with photon counting mode are shown in Fig. 3. The integration time for these signals is $15 \mathrm{~min}$ (26400 shots) and the data are binned with range resolution of $210 \mathrm{~m}$. This data was obtained during the night of Aug. 12, 2010. The laser pulse energy for the measurement was $300 \mathrm{~mJ}$. The atmospheric back-scattering ratio is taken into the inversion algorithm of line of sight wind speed (LOS) to minimize the aerosol effect in the lower altitude [12]. In Fig. 3 (a) the intensities corresponding to the two edge channel signals are almost equal between $25 \mathrm{~km}$ and $35 \mathrm{~km}$ according to the weak LOS wind speed. However, there is an obvious difference between $7 \mathrm{~km}$ and $25 \mathrm{~km}$, with a peak at 15

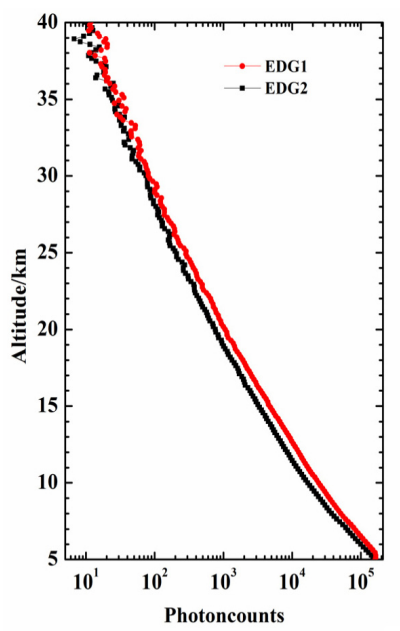

(a)

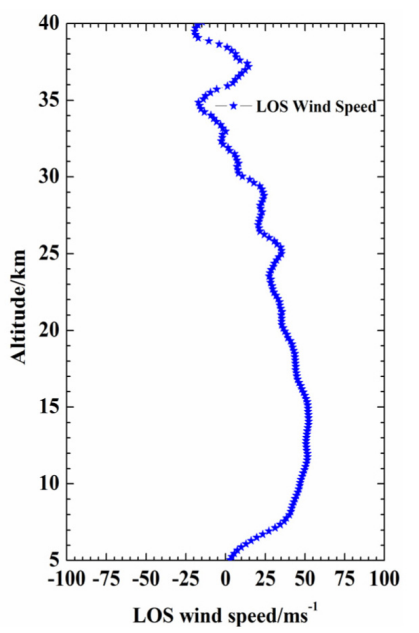

(b)
FIG. 3. (a) Photon counts detected in the molecular receiver for the two edge channels, EDG1 and EDG2, with range resolution of $210 \mathrm{~m}$ and shot number of 26400. (b) Los wind speed profile. $\mathrm{km}$ due to a large line-of-sight speed as shown Fig. 3 (b), in which the LOS velocity increases along the altitude from $5 \mathrm{~km}$ to $14 \mathrm{~km}$ with a peaking velocity of $52 \mathrm{~m} / \mathrm{s}$ at $14 \mathrm{~km}$. The horizontal wind velocity and direction can be obtained through vector computing from four line-of-sight wind velocities with an orientation azimuth interval of 90 degrees. The intercomparision experiment with rawinsonde was carried out on Aug. 14, 2010, as shown in Fig. 4. The results show a good agreement. A strong jet with peak horizontal wind speed of around $45 \mathrm{~m} / \mathrm{s}$ is clearly observed at $8 \mathrm{~km}$ for both instruments.

Following the testing and calibration experiment, we made continuous wind field observation in August 2010. Several valid wind data from August 2010 are drawn on one picture, as shown in the Fig. 5. Stratospheric quasi-zero wind layer can be found at around $20 \mathrm{~km}$ with a direction change from east to west evident. The low-level jet can be observed between the altitude $7 \mathrm{~km}$ and $15 \mathrm{~km}$ with the horizontal wind velocity around of $45 \mathrm{~m} / \mathrm{s}$ from the Aug. 10 to Aug.
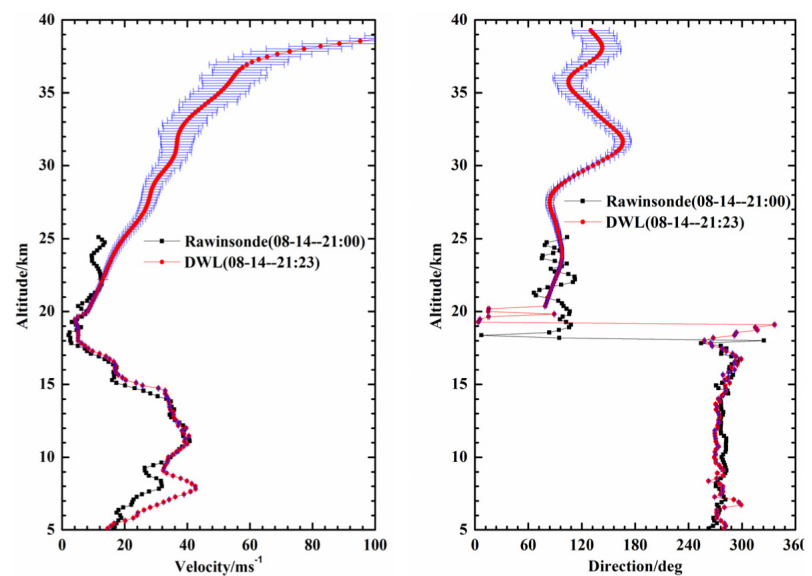

FIG. 4. The wind LIDAR intercomparision with rawinsonde.

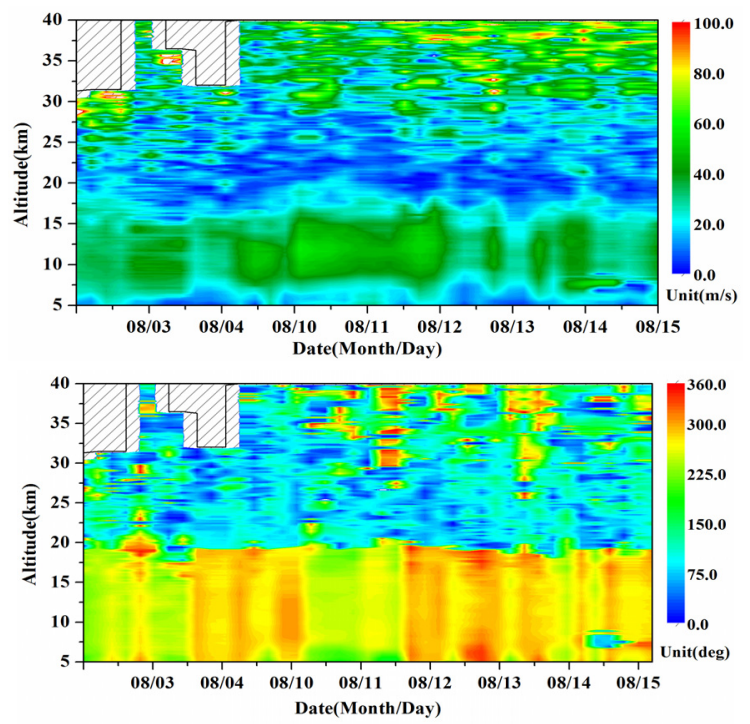

FIG. 5. The valid wind data of Aug. 2010. 
15. The wind velocities for the entire dataset almost increase along with the altitude, peaking at around $14 \mathrm{~km}$, then they decrease to a minimum at around $20 \mathrm{~km}$. They increase again from $20 \mathrm{~km}$ to $40 \mathrm{~km}$. The wind velocities show slight fluctuation above $30 \mathrm{~km}$ because of low signal-tonoise ratio. The wind directions are shown to be quasiwesterly below $20 \mathrm{~km}$ and quasi-easterly above $20 \mathrm{~km}$ in Fig. 5.

\section{CONCLUSION}

The principle of wind measurement and the mobile Rayleigh Doppler wind LIDAR system were described. This instrument can measure the wind field at altitudes from 5 $\mathrm{km}$ to $40 \mathrm{~km}$. An intercomparsion of wind velocity and direction was made between the mobile Rayleigh Doppler wind LIDAR measurements and rawinsonde measurements with good agreement. A continuous wind field observation experiment was carried out in Aug. 2010. A stratospheric quasi-zero wind layer can be found at around $20 \mathrm{~km}$ with a direction change from east to west evident. The wind field change process can be detected to help us investigate the atmospheric dynamics.

\section{ACKNOWLEDGMENT}

This work was supported by the Chinese Academy of Sciences (KZZD-EW-01-1) and National Natural Science Foundation of China (NSFC) project No. 41174130 and No. 41174131.

\section{REFERENCES}

1. M. L. Chanin, A. Garnier, A. Hauchecorne, and J. Porteneuve, "A Doppler LIDAR for measuring winds in the middle atmosphere," Geophys. Res. Lett. 16, 1273-1276 (1989).

2. D. Rees and I. S. McDermid, "Doppler LIDAR atmospheric wind sensor: re-evaluation of the $355 \mathrm{~nm}$ incoherent Doppler LIDAR," Appl. Opt. 29, 4133-4144 (1990).

3. B. Gentry, H. Chen, and D. Starr, "Profiling tropospheric winds with the Goddard LIDAR observatory for winds (GLOW)," in Proc. The 21st International Laser Radar Conference (Quebec, Canada, Jul. 2002), pp. 8-12.

4. W. E. Baker, G. D. Emmitt, F. Robertson, R. M. Atlas, J. E. Molinari, D. A. Bowdle, J. Paegle, and J. McElroy, "LIDAR-measured winds from space: a key component for weather and climate prediction," Bull. Amer. Meteor. Soc. 76, 869-888 (1995).

5. D. Sun, Z. Zhong, J. Zhou, H. Hu, and T. Kobayashi, "Accuracy analysis of the Fabry-Perot etalon based Doppler wind LIDAR," Opt. Rev. 12, 409-414 (2005).

6. H. Xia, D. Sun, Y. Yang, F. Shen, J. Dong, and T. Kobayashi, "Fabry-Perot interferometer based Mie Doppler LIDAR for low tropospheric wind observation," Appl. Opt. 46, 7120-7130 (2007).

7. F. Shen, H. Cha, D. Sun, D, Kim, and S. O. Kwon, "Low tropospheric wind measurement with Mie Doppler LIDAR," Opt. Rev. 15, 204-209 (2008).

8. I.-K. Song, G.-Y. Kim, S.-H. Baik, S.-K. Park, H.-K. Cha, S.-C. Choi, C.-M. Chung, and D.-H. Kim, "Measurement of aerosol parameters with altitude by using two wavelength rotational Raman signals," J. Opt. Soc. Korea 14, 221-227 (2010)

9. Z. Shu, L. Tang, G. Wang, D. Hu, D, Sun, and X. Dou, "Application of triple Fabry-Perot etalon for rayleigh wind LIDAR," Infrared and Laser Engineering 40, 1474-1480 (in Chinese) (2011).

10. F. Shen, H. Cha, J. Dong, D. Kim, D. Sun, and S. O. Kwon, "Design and performance simulation of a molecular Doppler wind LIDAR," Chinese Optics Letter 7, 593-597 (2009).

11. Z. Shu, L. Tang, J. Dong, F. Shen, D. Sun, X. Dou, and H.-K. Cha, "Performance of the triple Fabry-Perot etalon for wind LIDAR," Acta Optica Sinica 30, 1332-1336 (in Chinese) (2010).

12. F. Shen, Z. Shu, D. Sun, Z. Wang, X. Xue, T. Chen, and $X$. Dou, "Improvement of wind retrieval algorithm for Rayleigh Doppler LIDAR,” Acta. Phys. Sin. 61, 030702-1 030702-7 (in Chinese) (2012). 